\title{
Multivariate Statistical Analysis of Atom Probe Tomography Data
}

\author{
M.R. Keenan ${ }^{1}$, V.S. Smentkowski ${ }^{2}$, R.M. Ulfig ${ }^{3}$, E. Oltman ${ }^{3}$, D. J. Larson ${ }^{3}$, and T.F. Kelly ${ }^{3}$ \\ ${ }^{1} 8346$ Roney Road, Wolcott, NY 14590 \\ ${ }^{2}$ General Electric Corporate Research and Development, Niskayuna, NY 12309 \\ ${ }^{3}$ Imago Scientific Instruments Corporation, 5500 Nobel Drive, Madison, WI 53711 USA
}

Multivariate statistical analysis (MVSA) methods have been shown to yield many advantages in analytical instrumentation techniques because they offer an objective means to find complex information correlations in data that are not obvious to an observer and they maximize the discrimination of low-level signals from noise. Because they are objective in nature, the methods hold promise for automated data mining, especially in large data sets. Atom probe tomography (APT) hardware improvements in the past decade have shifted the bottleneck from data collection to data analysis. It is imperative, therefore, that methods are developed to automate analysis of the entire APT data set. MVSA has been applied successfully to other types of large 3D tomographic data sets including EDS [1] and ToF-SIMS [2]. We report, here, the adaptation of those techniques, in particular multivariate curve resolution (MCR), to APT data.

Analyzing APT data with MVSA techniques poses a number of challenges. Unlike typical analytical images, there is no "natural" voxel size as the volumes probed correspond to individual atoms. Careful attention must be paid to how the data are aggregated prior to MVSA to optimize the tradeoff between spatial resolution and sensitivity. Raw APT data also contain non-specific background signal, and when binned, are multinomially distributed due to compositional inhomogeneity on the nanometer scale. Properly accounting for these types of noise is critical for achieving good results with MSVA. Finally, the presence of multiple charge states corresponding to single atomic species requires analysis at higher mass spectral resolution leading to yet larger data sets. In the current work, we use improved numerical algorithms that scale better with size yielding substantial performance gains over tools previously used to analyze large data sets $[1,2]$.

A model system was simulated to validate our MVSA approach. A 97:3 Si:FeCu matrix with spherical $\mathrm{FeCu}$ precipitates of $4 \mathrm{~nm}$ diameter was created as shown in Fig. 1. While not meant to simulate a real material, the model captures the salient features of real APT data: spatial and spectral overlap, and multinomially distributed ions. This allows us to test the method against a known answer, and the MCR-derived components correctly identified both phases. In a second example, a multiphase nickel-based superalloy was analyzed as shown in Fig. 2. The alloy phases were readily discovered by the analysis and significant carbon segregation (green) was found at the interphase boundary. The last example is a polycrystalline silicon specimen. As shown in Fig. 3, P segregation to the grain boundary is just discernable in the atom map with a signal-to-noise ratio of about 2 . MVSA is able to clearly identify the voxels with elevated P content. Interestingly, with standard Poisson weighting [3] MCR cannot discover this component at the same level of spatial aggregation.

We are demonstrating for the first time that MVSA has several important applications to APT data. We can envision fully automated analyses that report phase statistics for size, orientation, composition, segregation, and volume fraction. These algorithms are fast and run on a desktop computer in seconds. Commercial versions of these algorithms in data analysis routines are planned. 


\section{References}

[1] P.G. Kotula, M.R.Keenan and J.R.Michael, Microsc. Microanal. 12 (2006) 36.

[2] V.S. Smentkowski, et.al., Anal. Chem. 79 (2007) 7719.

[3] M.R. Keenan and P.G. Kotula, Surf. Interface Anal. 36 (2004) 203.
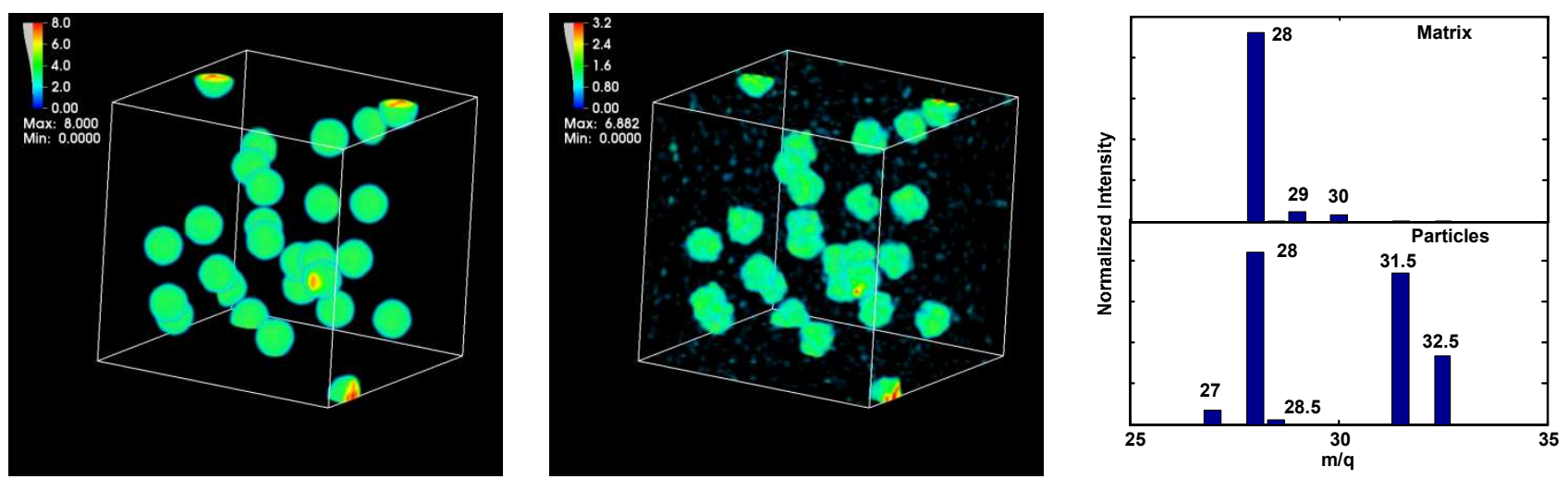

FIG. 1. $4 \mathrm{~nm} \mathrm{FeCu}$ particles in a 97:3 $\mathrm{Si}: \mathrm{FeCu}$ matrix at $0.5 \mathrm{~nm}$ resolution. a) model, b) MCRestimated abundances for the particle component; detector efficiency was $37 \%$. c) MCR component mass spectra.
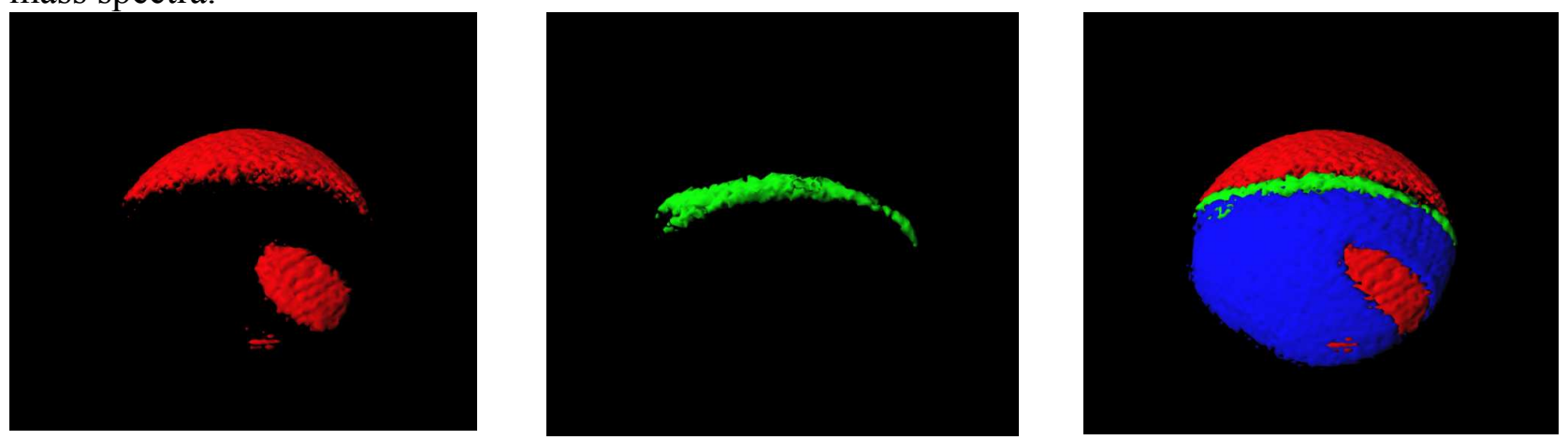

FIG. 2. Nickel-based superalloy a) Component 1 is a nickel-rich phase. b) Component 2 is a carbonrich region at the interphase boundary. c) Two-phase image showing segregation at the boundary.
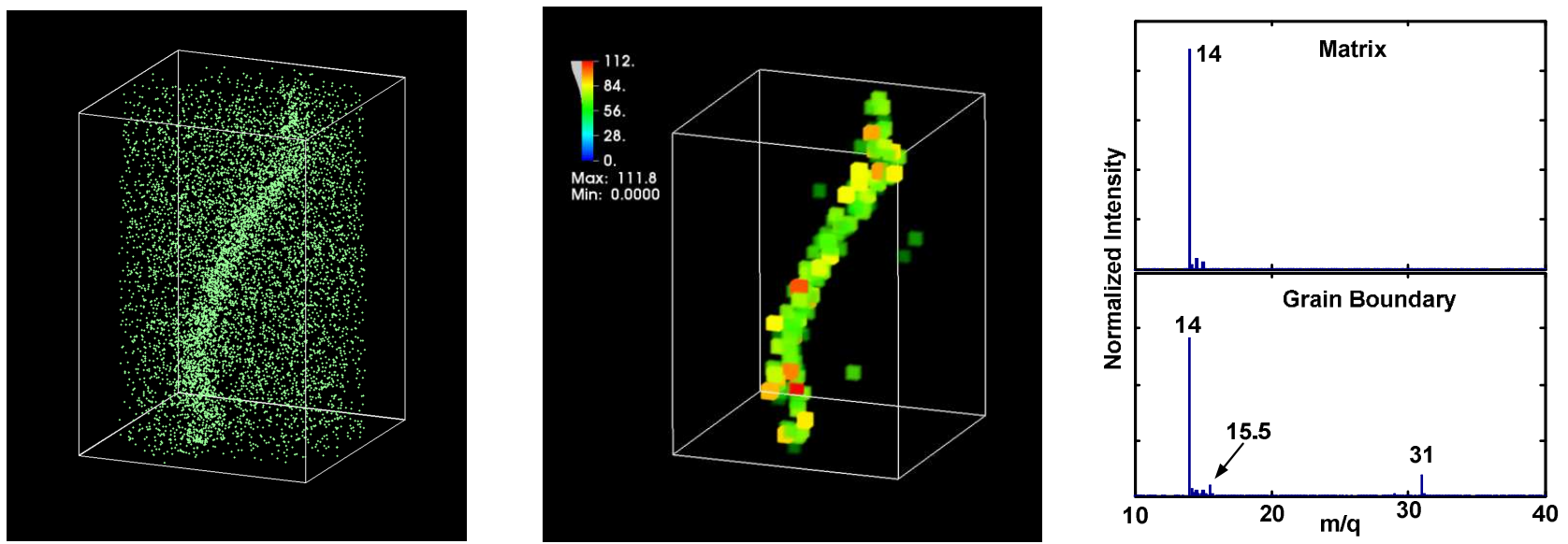

FIG. 3. Polysilicon doped with phosphorus. a) Phosphorus atom map. b) MCR-derived abundances for the phosphorus-containing component showing the grain boundary. c) Component mass spectra. 